illustration of this phenomenon we give the following example of an ideal with a basis which is a subset of its basic set. ${ }^{10}$

Let $\mathcal{F}$ be the field of all rational functions of $x$ with transforming defined as the operation of replacing $x$ by $x+1$. We consider the polynomials

$$
y_{1}-y, \quad z^{2}-y, \quad z_{1}-z .
$$

Using Theorem IX of M.D.P. one shows easily that (2) is a basic set of a prime reflexive ideal $\Lambda$ with coefficients in $\mathcal{F}$. Since the initials of the polynomials of (2) are unity, it follows that it is a basis for $\Lambda$. But the equations $z_{1}-z=0, z^{2}-y=0$, imply that $y_{1}=z_{1}^{2}=z^{2}=y$; so that any solution of these equations is also a solution of $y_{1}-y=0$. Thus $z^{2}-y, z_{1}-z$ is itself a basis for $\Lambda$.

RUTGERS UNIVERSITY

${ }^{10}$ This example emerged during a conversation of the author with E. R. Kolchin.

\title{
INVERSIVE DIFFERENCE FIELDS
}

\section{RICHARD M. COHN}

It may happen that the functions of an analytic difference field admit not only the operation of replacing $z$ by $z+1$, but also its inverse. Since these two operations have essentially the same properties it is to be expected that to each theorem concerning difference equations there will be a corresponding theorem, valid in fields of the type we have just described, in which the rôles of the highest and lowest transforms of the unknowns are interchanged. For example, J. F. Ritt has shown ${ }^{1}$ that the number of ordinary manifolds of a firstorder difference polynomial in the unknown $y$ does not exceed its degree in $y_{1}$; and he observes that in fields where the inverse substitution is always possible, the number of ordinary manifolds is also limited by the degree in $y$.

The study of abstract difference fields enables us to apply this principle in every case; for, as we shall show, every abstract difference field can be extended to a difference field in which there exists, for every element $h$ of the field, an element $g$ such that $h$ is the trans-

Received by the editors April 29, 1948.

${ }^{1}$ J. F. Ritt, Algebraic difference equations, Bull. Amer. Math. Soc. vol. 40 (1934) pp. 303-308. 
form of $g$. We shall call a field with this property an inversive difference field.

The first part of this note, then, will be devoted to the brief proof that every difference field has an inversive extension. In the second part we apply this theorem to complete certain earlier results on the structure of the manifold of a difference polynomial.

\section{PART I}

THEOREM. Every difference field may be imbedded in an inversive difference field.

We first prove that any difference field $F$ may be imbedded in a field $F^{\prime}$ isomorphic to $F$ and such that every element of $F$ is a transform of an element of $F^{\prime}$. Let $F_{1}$ be the set consisting of first transforms of elements of $F$. It is easily seen that $F_{1}$ is a difference field isomorphic to $F$ by the correspondence which maps any element $\alpha$ of $F$ on its transform $\alpha_{1}$. Now we may extend ${ }^{2}$ this isomorphism to an isomorphism between the extension $F$ of $F_{1}$, and an extension $F^{\prime}$ of $F$. Then $F^{\prime}$ has the desired properties.

Let us now construct, by this process, a sequence of fields $F, F^{\prime}$, $F^{\prime \prime}, \cdots$, such that $F^{(k)}$ is isomorphic to $F^{(k+1)}$, and every element of $F^{(k)}$ is a transform of an element of $F^{(k+1)}$. Let $\bar{F}$ be the difference field which is the union of the $F^{(k)}, k=0,1,2, \cdots$. Then $\bar{F}$ is evidently the inversive extension of $F$ required by the theorem.

\section{PART II}

We have shown elsewhere ${ }^{3}$ that the essential irreducible manifolds of a difference polynomial $A$ in unknowns $y_{1}, \cdots, y_{n}$ may be divided into ordinary and essential singular manifolds. No ordinary manifold annuls a polynomial of lower effective order than $A$ in any $y_{k}$, while each singular manifold of $A$ annuls such a polynomial for every $y_{k}$, $1 \leqq k \leqq n$. Concerning these two types of manifolds we may make the following statements:

(1) There is always at least one ordinary manifold; but their number does not exceed the degree of $A$ in the lowest, nor in the highest transform of any $y_{i}$ which appears effectively.

${ }^{2}$ Let $H$ be isomorphic to, but contain no element in common with, $F$. Let $H_{1}$ be the subfield of $H$ corresponding to $F_{1}$. By the obvious extension to difference fields of a theorem in van der Waerden, Moderne Algebra, 2d ed., New York, F. Ungar, p. 42 , the extension $F^{\prime}$ of $F$ exists isomorphic to $H$, with $H_{1}$ corresponding to $F$ under the isomorphism. Evidently $F^{\prime}$ has the properties we require.

${ }^{3}$ A note on the singular manifolds of a difference polynomial, Bull. Amer. Math. Soc. vol. 54 (1948) pp. 917-922. This note is referred to henceforth as S.M. 
(2) The essential singular manifolds of $A$ annul its formal first partial derivatives with respect to the lowest and with respect to the highest transforms of each $y_{i}$ which appear effectively.

Except for the reference to transforms of lowest order these statements have been proved previously. ${ }^{4}$ We now complete the proof in this respect.

We consider an inversive difference field $F$, and denote by $F^{*}$ the difference field consisting of the elements of $F$ with the operation of transforming defined as the inverse of that in $F$. Let $A$ be any polynomial in unknowns $y, y_{1}, \cdots, y_{n}$, with coefficients in $F$. Then we denote by $A^{*}$ a polynomial obtained from $A$ by replacing each $y_{i k}$ appearing in $A$ by $y_{i, m-k}^{\prime}$, where $m$ is an integer sufficiently large so that $m-k$ is always non-negative. In other respects $A$ is unaltered except that its coefficients are now to be regarded as elements of $F^{*}$ rather than $F$. The operation * will be applied in what follows to only a finite number of polynomials at any one time. We may therefore always choose an $m$ which can be used for all the polynomials under consideration, and we shall assume in each case that this has been done.

We prove a lemma concerning the operation *.

Lemma 1. Let $F$ be an inversive difference field, and let $B$ and $A$ be polynomials in $F$. Then $B$ holds $A$ if, and only if, $B^{*}$ holds $A^{*}$.

We assume first that $B^{*}$ holds $A^{*}$, but that $A$ has a solution $y_{i}=\alpha_{i}$, in an extension $G$ of $F$, which does not annul $B$.

Let $\bar{G}$ be an inversive extension of $G$, and let $\bar{G}^{*}$ be the field consisting of the elements of $\bar{G}$ with the transforming operation defined as the inverse of that of $\bar{G}$. Then $\bar{G}^{*}$ is an extension of $F^{*}$. The $\alpha_{i}$ lie in $\bar{G}^{*}$, but in this field $\alpha_{i k}$ is the transform of $\alpha_{i, k+1}$ if $k \geqq 0$, and transforms of the $\alpha_{i 0}$ exist. When we substitute the $\alpha_{i k}$ for the $y_{i, m-k}^{\prime}$, where $m$ is the integer used in defining ${ }^{*}$, it is evident that $A^{*}$ vanishes while $B^{*}$ does not. This contradicts the statement that $B^{*}$ holds $A^{*}$. Thus the implication is proved in one direction.

We next assume that $B$ holds $A$, but that $A^{*}$ has a solution $y_{i}^{\prime}=\beta_{i}$ in an extension $G^{*}$ of $F^{*}$, which does not annul $B^{*}$. Let $\bar{G}^{*}$ be an inversive extension of $G^{*}$, and let $\bar{G}$ be the field consisting of the elements of $\bar{G}^{*}$, with the transforming operation defined as the inverse of that in $\bar{G}^{*}$. Then $\bar{G}$ is an extension of $F$. When we replace each $y_{i k}$ by $\beta_{i, m-k}$, where $m$ is the integer used in defining ${ }^{*}$, it is evident that $A$ vanishes, while $B$ does not. This is a contradiction which estab-

4 Manifolds of difference polynomials, Trans. Amer. Math. Soc. vol. 64 (1948) pp. 133-172 and S.M. 
lishes the remaining implication. Thus the lemma is proved.

It is convenient to prove the next two lemmas together. We retain the notation of Lemma 1 . In a previous paper ${ }^{5}$ we defined the $y_{i}$ separant of a difference polynomial as its formal first partial derivative with respect to the highest transform of $y_{i}$ present. We now introduce the term inverse $y_{i}$-separant for the partial derivative with respect to the lowest transform of $y_{i}$ present. We may now state the lemmas.

Lemma 2. The polynomials $A$ and $A^{*}$ have the same number of ordinary manifolds.

Lemma 3. The essential singular manifolds of $A$ annul its inverse separants. (Note that the coefficient field $F$ is still assumed to be inversive.)

Let $M$ be any essential irreducible manifold of $A$, and let $\Lambda$ be the prime ideal consisting of all polynomials with coefficients in $F$ which are annulled by all solutions in $M$. Let $G$ be the field which results from $F$ by adjoining the general point $y_{i}=\alpha_{i}$ of $\Lambda$. Let $\bar{G}$ denote the inversive extension of $G$ formed as in Part I, and finally let $\bar{G}^{*}$ be the field consisting of the elements of $\bar{G}$, but with the inverse transforming operation. Then $\bar{G}^{*}$ is an extension of $F^{*}$.

The substitution $y_{i k}^{\prime}=\alpha_{i, m-k}$ annuls $A^{*}$. We denote by $\Lambda^{*}$ the reflexive prime ideal consisting of all polynomials with coefficients in $F^{*}$ which vanish when these values, or their appropriate transforms, are assigned to the $y_{i k}^{\prime}$.

Let $T$ be any polynomial with coefficients in $F^{*}$. We shall investigate under what circumstances $T$ is in $\Lambda^{*}$.

By an operation similar to ${ }^{*}$ we convert $T$ into a polynomial $T^{\prime}$. Specifically, we replace each $y_{t \mathrm{k}}^{\prime}$ by $y_{i, p-k}^{\prime \prime}$, where $p$ is a sufficiently great integer so that the subscripts are all non-negative, and we consider the coefficients of the resulting polynomial as elements of $F$. The substitutions $y_{i, k+p-m}^{\prime \prime}=\alpha_{i, k}$ annul $T^{\prime}$ if and only if $T$ holds $\Lambda^{*}$. In this substitution the $\alpha_{i k}$ with $k$ negative are to be understood as the corresponding positive transforms of $\alpha_{i 0}$ in $F^{*}$.

Let $\bar{T}$ be formed by replacing $y_{i, k+p-m}^{\prime \prime}$ by $y_{i k}$ in a transform of $T^{\prime}$ which is such that only non-negative values of $k$ appear. Then $T$ holds $\Lambda^{*}$ if and only if $\bar{T}$ is annulled by the substitution $y_{i k}=\alpha_{i k}$, in other words, if and only if $\bar{T}$ is in $\Lambda$.

To prove Lemma 2 we let $M$ be an ordinary manifold of $A$. We claim that the manifold of $\Lambda^{*}$ is an ordinary manifold of $A^{*}$. For, if it

${ }^{5}$ S.M., $\$ 1$. 
is not, there is a $T$ of effective order less than that of $A^{*}$ in $y_{1}$ which holds $\Lambda^{*}$. Then the corresponding $\bar{T}$ holds $\Lambda$. Since $\bar{T}$ is obviously of effective order less than $A$ in $y_{1}$ this is impossible, so that our statement is justified. Thus there corresponds to each ordinary manifold of $A$ an ordinary manifold of $A^{*}$.

Let $M_{1}, M_{2}$ be two distinct ordinary manifolds of $A$ (if such exist), and $\Lambda_{1}, \Lambda_{2}$ the corresponding prime ideals. We form $\Lambda_{1}{ }^{*}, \Lambda_{2}{ }^{*}$ from $\Lambda_{1}, \Lambda_{2}$ as $\Lambda^{*}$ was formed from $\Lambda$. Then $\Lambda_{1}^{*}, \Lambda_{2}{ }^{*}$ are distinct for large $m$. For let $C$ be in $\Lambda_{1}$ but not in $\Lambda_{2}$. If the $m$ used in defining ${ }^{*}$ is sufficiently large we may form $C^{*}$. Then $C^{*}$ holds $\Lambda_{1}{ }^{*}$ but not $\Lambda_{2}{ }^{*}$, so that these are distinct. It follows that, for large $m, A^{*}$ has at least as many ordinary manifolds as $A$. It remains to prove this statement for all $m$.

We consider integers $m_{1}$ and $m_{2}>m_{1}$. Let us use * to denote specifically the operator previously so designated, but with $m$ fixed as $m_{1}$, and let us use ${ }^{* *}$ to denote the corresponding operator with $m=m_{2}$. Then $A^{* *}$ results from $A^{*}$ by replacing each $y_{i j}^{\prime}$ by its transform of order $m_{2}-m_{1}$. Let $A^{\prime}$ denote the $\left(m_{2}-m_{1}\right)$ th transform of $A^{*}$. Then $A^{\prime}$ and $A^{*}$ have the same number of ordinary manifolds. Consider the isomorphism which carries $F^{*}$ into the field $F^{\prime}$ consisting of the transforms of order $m_{2}-m_{1}$ of elements of $F^{*}$. This isomorphism carries $A^{* *}$ into $A^{\prime}$. Thus $A^{* *}$ and $A^{\prime}$ have the same number of ordinary manifolds. We conclude that $A^{* *}$ and $A^{*}$ have the same number of ordinary manifolds. Now by choosing $m_{2}$ sufficiently large we may show, by the method of the preceding paragraph, that $A^{* *}$ possesses at least as many ordinary manifolds as $A$. Then this is also true of $A^{*}$.

Now if we let $T=A^{*}$, and form $\bar{T}$ as above, we see, by an argument similar to that of the preceding paragraphs, that $\bar{T}$ possesses at least as many ordinary manifolds as $A^{*}$. But, if we choose $p=m$, we find that $\bar{T}=A$. Combining this result with that of the preceding paragraph we verify Lemma 2 .

We now turn to the proof of Lemma 3. We let $M$ denote an essential singular manifold of $A$, and form its prime ideal $\Lambda$ and the corresponding prime ideal $\Lambda^{*}$ of $A^{*}$ as at the beginning of the discussion of Lemma 2. Let $M_{1}, M_{2}, \cdots, M_{r}$ denote the ordinary manifolds of $A, \Lambda_{1}, \Lambda_{2}, \cdots, \Lambda_{r}$ their corresponding prime ideals, and $\Lambda_{1}{ }^{*}, \Lambda_{2}{ }^{*}, \cdots$, $\Lambda_{r}^{*}$ the prime ideals of $A^{*}$ formed from the $\Lambda_{i}$. The proof of Lemma 2 shows that, at least for large $m$, the $\Lambda_{i}{ }^{*}$ are distinct and their manifolds constitute the totality of ordinary manifolds of $A^{*}$. Let $C_{i}$, $i=1, \cdots, r$, be a polynomial of $\Lambda_{i}$ which does not hold $\Lambda$. We may assume that $m$ is large enough so that we can form the $C_{i}{ }^{*}$, $i=1, \cdots, r$. Each $C_{i}{ }^{*}$ is in $\Lambda_{i}{ }^{*}$ but not in $\Lambda^{*}$. It follows that the mani- 
fold of $\Lambda^{*}$ is contained within no ordinary manifold of $A^{*}$. Then any essential irreducible manifold of $A^{*}$ which contains the manifold of $\Lambda^{*}$ is an essential singular manifold, and therefore annuls each $y_{i}$-separant of $A^{*}$. We denote the $y_{1}$-separant by $T$, and form $\bar{T}$ as at the beginning of the proof of Lemma 2, using $p=m$. By what precedes $\bar{T}$ is annulled by a general point of $\Lambda$, so that it holds $\Lambda$. But $\bar{T}$ is the inverse $y_{1}$-separant of $A$, and we may prove similarly that each inverse $y_{i}$-separant holds $\Lambda$. Thus the lemma is proved.

We now complete the proofs of statements (1) and (2) at the beginning of Part II. For inversive fields the previously unproved part of statement (1) is an immediate consequence of Lemma 2. Now let $F$ be any difference field, and $A$ an algebraically irreducible difference polynomial with coefficients in $F$. Let $M_{1}, M_{2}, \cdots, M_{r}$ be the ordinary manifolds of $A$, and $\Lambda_{1}, \Lambda_{2}, \cdots, \Lambda_{r}$ the corresponding prime ideals. We let $y_{i}=\alpha_{i}$ be a general point of $\Lambda_{1}$ and denote by $G_{1}$ the field obtained by adjoining the $\alpha_{i}$ to $F$. In a similar way we form fields $G_{i}$ containing general points of the remaining $\Lambda_{i}$.

There exists, by the theorem of Part I, an inversive extension $\bar{G}_{i}$ of $G_{i}, i=1, \cdots, r$. The elements of any $\bar{G}_{i}$ whose transforms of any order lie in $F$ form a subfield $\bar{F}_{i}$. Now it is easy to see that the $\bar{F}_{i}$ are all isomorphic. We denote any one of them by $\bar{F}$. It is possible to construct, ${ }^{6}$ for each $i$, an extension of $\bar{F}$ isomorphic to $\bar{G}_{i}$ and therefore containing a general point of $\Lambda_{i}$. We shall assume that the $\bar{G}_{i}$ above are actually extensions of the inversive field $\bar{F}$.

Let $\Sigma_{1}$ be the reflexive prime ideal consisting of those polynomials with coefficients in $\bar{F}$ which are annulled by the substitution $y_{i}=\alpha_{i}$. $\Sigma_{1}$ must be the prime ideal corresponding to an ordinary manifold of $A$ considered as a polynomial with coefficients in $\bar{F}$, or of an irreducible factor of $A$ in $\bar{F}$ which is of the same effective order as $A$. For otherwise some polynomial $D$ of $\Sigma_{1}$ is of lower effective order than $A$ in some $y_{i}$, say $y_{1}$. $A$ transform, $D_{k}$, of $D$ has coefficients in $F$. Then $D_{k}$ is in $\Lambda_{1}$, since it is annulled by the substitution of the $\alpha_{i}$ for the $y_{i}$, and is of lower effective order than $A$ in $y_{1}$. This is impossible, so that our statement is verified.

We now construct $\Sigma_{i}, i=2, \cdots, r$, corresponding to each $\Lambda_{i}$. The construction can be carried out similarly to that of $\Sigma_{1}$. The $\Sigma_{i}$ are all prime ideals corresponding to ordinary manifolds of irreducible factors of $A$ considered as a polynomial with coefficients in $\bar{F}$. Furthermore they are all distinct. For $\Lambda_{i}$ contains a polynomial $C$ which is not annulled by the general point of $\Lambda_{j}, j \neq i$. Then $C$ is in $\Sigma_{i}$, but not

\footnotetext{
${ }^{6}$ The method of footnote 2 is to be used.
} 
in $\Sigma_{j}$. Thus we see that there are at least as many ordinary manifolds of irreducible factors of $A$, considered as a polynomial in $\bar{F}$, as of $A$ considered as a polynomial in $F$. Then the truth of statement (1) for the inversive field $\bar{F}$ implies its truth for $F$.

We turn now to statement (2). Let $M$ be an essential singular manifold of the algebraically irreducible difference polynomial $A$ with coefficients in the field $F$, and let $\Lambda$ be the reflexive prime ideal consisting of all polynomials annulled by $M$. Let $y_{i}=\alpha_{i}$ be a general point of $\Lambda$. We form the inversive extension $\bar{F}$ of $F$ as in Part I. Let $\Sigma$ be the set of polynomials with coefficients in $\bar{F}$ which are annulled when the $y_{i}$ are replaced by the $\alpha_{i}$. Obviously $\Sigma$ is a reflexive prime ideal held by $A$. Let $N$ be an essential irreducible manifold of $A$ (as a polynomial in $\bar{F}$ ) which contains the manifold of $\Sigma$. Let $\psi$ be the prime ideal consisting of those polynomials with coefficients in $\bar{F}$ which are annulled by the solutions of $N$. We form the system $\psi^{\prime}$ consisting of those polynomials of $\psi$ with coefficients in $F . \psi^{\prime}$ is held by a polynomial of effective order in $y_{1}$ lower than that of $A$. For otherwise its manifold is an ordinary manifold of $A$ as a polynomial in $F$. But this is impossible because the general point $y_{i}=\alpha_{i}$ of the ideal $\Lambda$, whose manifold is an essential singular manifold, annuls $\psi^{\prime}$.

It follows that $N$ is an essential singular manifold of an irreducible factor of $A$ considered as a polynomial in $\bar{F}$. Lemma 3 shows that each inverse separant of this factor is in $\psi$. It follows that the inverse separants of $A$ vanish for the solution $y_{i}=\alpha_{i}$, and so are in $\Lambda$. This completes the proof of statement 2 .

RUTGERS UNIVERSITY 